\title{
Testing for Asymmetric Dependence
}

Citation for published version (APA):

Manner, H. (2008). Testing for Asymmetric Dependence. Maastricht University School of Business and Economics. METEOR Research Memorandum No. 042 https://doi.org/10.26481/umamet.2008042

Document status and date:

Published: 01/01/2008

DOI:

10.26481/umamet.2008042

Document Version:

Publisher's PDF, also known as Version of record

\section{Please check the document version of this publication:}

- A submitted manuscript is the version of the article upon submission and before peer-review. There can be important differences between the submitted version and the official published version of record.

People interested in the research are advised to contact the author for the final version of the publication, or visit the DOI to the publisher's website.

- The final author version and the galley proof are versions of the publication after peer review.

- The final published version features the final layout of the paper including the volume, issue and page numbers.

Link to publication

\footnotetext{
General rights rights.

- You may freely distribute the URL identifying the publication in the public portal. please follow below link for the End User Agreement:

www.umlib.nl/taverne-license

Take down policy

If you believe that this document breaches copyright please contact us at:

repository@maastrichtuniversity.nl

providing details and we will investigate your claim.
}

Copyright and moral rights for the publications made accessible in the public portal are retained by the authors and/or other copyright owners and it is a condition of accessing publications that users recognise and abide by the legal requirements associated with these

- Users may download and print one copy of any publication from the public portal for the purpose of private study or research.

- You may not further distribute the material or use it for any profit-making activity or commercial gain

If the publication is distributed under the terms of Article $25 \mathrm{fa}$ of the Dutch Copyright Act, indicated by the "Taverne" license above, 
Hans Manner

Testing for Asymmetric Dependence

$\mathrm{RM} / 08 / 042$

JEL code: C12, C22

\section{METE@R

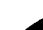

Maastricht research school of Economics

of TEchnology and ORganizations

Universiteit Maastricht

Faculty of Economics and Business Administration

P.O. Box 616

NL - 6200 MD Maastricht

phone : ++31433883830

fax $\quad$ : ++31433884873 


\title{
Testing for Asymmetric Dependence
}

\author{
Hans Manner*
}

June 30,2008

\begin{abstract}
We study how to measure and test for differences in dependence for small and large realizations of two variables of interest. We introduce a conditional version of Kendall's tau and provide formulas to evaluate it for any copula of interest. Two tests based on well known copulas are proposed to test the null hypothesis of symmetric dependence and these tests outperform the one proposed by Hong et al. (2007) in a Monte Carlo study. Additionally, we suggest three examples of data generating processes that can lead to asymmetric dependence and study these both analytically and in a Monte Carlo framework. Finally, we illustrate the use of our tests on stock market returns and on quarterly US GNP and Unemployment data and we find evidence of asymmetries and nonlinearities.
\end{abstract}

Keywords: Asymmetric dependence, Copulas, exceedance correlation, Kendall's tau, Copula Markov models.

JEL Classification: C12, C22

*Department of Quantitative Economics, Maastricht University, PoBOX 616, MD 6200, Maastricht, The Netherlands, Tel.: 003143 3883695, e-mail: h.manner@ke.unimaas.nl 


\section{Introduction}

Asymmetries in correlation of financial assets can have important implications for portfolio selection, hedging and pricing of options with multiple underlying assets. Several studies such as King and Wadhwani (1990), Longin and Solnik (1995) or Ramchand and Susmel (1998) have found that correlations increase during volatile periods. Such findings are supported by factor models for asset pricing, which imply that an increase in the volatility of the factors leads to higher correlations between different stocks or stock market indices. However, more recently Longin and Solnik (2001) noted that a "bear market, not volatility per se is the driving force in increasing correlation". Cappiello et al. (2005) and Candelon and Manner (2007) even found that high volatility may decrease dependence between financial markets. Ang and Chen (2002) propose a test whether a given model can explain the asymmetric correlation and find evidence of asymmetries in stock returns. Based on this work Hong et al. (2007) provide a model free test for the null hypothesis of symmetric correlation, provide the asymptotic distribution of their test statistic and demonstrate the economic significance of taking into account asymmetries in investment decisions. Another study focusing on asymmetric dependence structures is Cappiello et al. (2007) who extended the well known DCC model for correlations and find evidence of asymmetries in conditional correlations.

A tool that has been frequently used to model asymmetric dependencies are copulas, which are that part of a multivariate distribution function that fully describes the dependence between the variables of interest (see Nelson, 2007, for an introduction and e.g. Patton, 2006, for an application). Although many authors have noted that certain copulas imply asymmetric dependence this has not been formalized and tested for in the copula context. In this paper we try to bridge this gap. Instead of using linear correlation as our canonical measure of dependence we use the more robust Kendall's tau that has a number of desirable properties such as being invariant under monotone transformations of the underlying variables (see Joe, 1997). Our first contribution is to define a conditional version of Kendall's tau and we provide formulas that can be used to evaluate the conditional Kendall's tau for a given copula. This allows us to assess the degree of asymmetry for two well known and frequently used families of copulas, namely the Gumbel and the Clayton (or Cook-Johnson) families. Based on these two copulas we construct two simple likelihood ratio tests for the null hypothesis of symmetric dependence. In a Monte Carlo experiment we compare these tests to the one proposed by Hong et al. (2007) and we find that these tests are clearly superior in terms of power, especially for small samples. Next, we suggest three simple data generating processes that can cause asymmetric dependence. The first two are returns generated by bear and bull markets with different mean returns, variances and correlations over the

regimes, and data coming from a nonlinear VAR's, where ignoring the nonlinearity causes 
asymmetric dependence of the residuals. The third case makes use of the fact that copulas cannot only be used to model contemporaneous dependence of two (or more) variables, but that the class of Copula Markov Models, introduced by Joe (1997) and studied by Bouyé et al. (2001) and Chen and Fan (2006a), can be used to model dependence between a variable and its own lags. We show that data generated from a self exciting smooth transition autoregression (SETAR) can cause asymmetric dependence through time. These three cases are considered in a Monte Carlo study. Then we test for asymmetric dependencies in international stock market returns and reject the null of symmetric dependence for a majority of cases. Finally, we apply the technique to quarterly returns of US GNP and unemployment data, that have been modeled with a threshold VAR by Altissimo and Violante (2001). In the autoregressive dynamics we find evidence of asymmetric dependence for unemployment, but not for GNP. Next, the data are fit to both a linear VAR and the threshold VAR by Altissimo and Violante (2001) and we run our tests on the residuals. The residuals of the linear VAR show asymmetries, whereas those of the nonlinear model are characterized by symmetric dependence.

The rest of the paper is structured as follows. In section 2 the concept of asymmetric dependence is introduced, the tests by Ang and Chen(2002) and Hong et al. (2007) are reviewed and we propose our alternative tests. Section 3 provides examples of data generating processes that can cause asymmetric dependence and these examples are analyzed. In section 4 an extensive Monte Carlo study is provided that shows the behavior of the tests under different scenarios. In section 5 an empirical application on US macroeconomic data is provided and section 6 concludes.

\section{Testing for Asymmetric Dependence}

We introduce the concept of exceedance correlation and we propose a conditional version of Kendall's tau in this section. Based on this we study the dependence properties of two well known families of copulas and we propose two simple tests for the null hypothesis of symmetric dependence using these copulas.

\subsection{Asymmetric Dependence}

Let $X_{t}$ and $Y_{t}$, for $t=1, \ldots, T$, be two stationary stochastic processes (e.g. stock returns) and consider the exceedance correlation between the two variables as studied by Longin and Solnik (2001), Ang and Chen (2002) and Hong et al. (2007). The exceedance correlation at 
level $c$ is defined as

$$
\begin{aligned}
& \rho^{+}(c)=\operatorname{corr}\left(X_{t}, Y_{t} \mid X_{t}>c, Y_{t}>c\right) \\
& \rho^{-}(c)=\operatorname{corr}\left(X_{t}, Y_{t} \mid X_{t}<-c, Y_{t}<-c\right) .
\end{aligned}
$$

The null hypothesis of symmetric correlation then is

$$
H_{0}: \quad \rho^{+}(c)=\rho^{-}(c), \quad \forall c \geq 0
$$

against

$$
H_{1}: \quad \rho^{+}(c) \neq \rho^{-}(c), \quad \text { for some } c \geq 0 .
$$

Denote the estimates of the exceedance correlation by $\hat{\rho}^{+}(c)$ and $\hat{\rho}^{-}(c)$ and let $\rho(c, \phi)$ be the exceedance correlation implied by a given model with parameter $\phi$. Then the test statistic proposed by Ang and Chen (2002) is given by

$$
H^{2}=\sum_{i=1}^{m} w\left(c_{i}\right)\left(\rho\left(c_{i}, \phi\right)-\hat{\rho}^{+}\left(c_{i}\right)\right)^{2},
$$

where $c_{1}, \ldots, c_{m}$ are $m$ exceedance levels and $w\left(c_{i}\right)$ is a weight. $\hat{\rho}^{+}(c)$ may be replaced by $\hat{\rho}^{-}(c)$ in the formula. Hong el al. (2007) derive the asymptotic distribution of $H^{2}$, which is a mixture of $m$ independent chi-squared random variables with weights equal to the eigenvalues of the weighted covariance matrix of $\hat{\rho}$. This test can be used to test whether a given model can explain the empirical exceedance correlation.

The test proposed by Hong el al. (2007) is model free and can be used to test the null hypothesis given in (3). For

$$
\hat{\rho}^{+}-\hat{\rho}^{-}=\left[\hat{\rho}^{+}\left(c_{1}\right)-\hat{\rho}^{-}\left(c_{1}\right), \ldots, \hat{\rho}^{+}\left(c_{m}\right)-\hat{\rho}^{-}\left(c_{m}\right)\right]^{\prime}
$$

and $T$ the sample size their test statistic is

$$
J_{\rho}=T\left(\hat{\rho}^{+}-\hat{\rho}^{-}\right)^{\prime} \hat{\Omega}^{-1}\left(\hat{\rho}^{+}-\hat{\rho}^{-}\right),
$$

where $\hat{\Omega}$ is a heteroscedasticity and autocorrelation (HAC) robust covariance estimator like the one proposed by Newey and West (1994). This statistic is shown to asymptotically follow a chi-square distribution with $m$ degrees of freedom.

Both approaches measure dependence through the correlation coefficient. As a measure of dependence, however, linear correlation is only appropriate when the data follows an elliptical distribution. For drawbacks of using linear correlation for modeling dependence 
see Embrechts el al. (2002).

As an alternative we suggest using the concept of concordance for measuring dependence. Consider two pairs of observations $\left(x_{i}, y_{i}\right)$ and $\left(x_{j}, y_{j}\right)$ from $\left(X_{t}, Y_{t}\right)$. We call these pairs of observations concordant if $\left(x_{i}-x_{j}\right)\left(y_{i}-y_{j}\right)>0$ and discordant if $\left(x_{i}-x_{j}\right)\left(y_{i}-y_{j}\right)<0$. Hence, two random variables are said to be concordant, when large values of one random variable tend to be associated with large values of the other, and similarly small values tend to be associated with each other.

Using the concept of concordance we are now able to introduce a measure of association known as Kendall's tau. Its sample version is defined as the fraction of concordant pairs of observations in the sample minus the fraction of discordant pairs of observations. The population version of Kendall's tau is defined as the difference between the probability of concordance and the probability of discordance.

$$
\tau=\tau\left(X_{t}, Y_{t}\right)=P\left[\left(X_{i}-X_{j}\right)\left(Y_{i}-Y_{j}\right)>0\right]-P\left[\left(X_{i}-X_{j}\right)\left(Y_{i}-Y_{j}\right)<0\right] \text { for } i \neq j .
$$

Since Kendall's tau is only based on the ranks of the observations it is, unlike the correlation coefficient, invariant under monotone transformations of $X_{t}$ and $Y_{t}$. Therefore, consider the random variables $U_{t}=F\left(X_{t}\right)$ and $V_{t}=G\left(Y_{t}\right)$, where $F$ and $G$ are the (unconditional) marginal distributions of $X_{t}$ and $Y_{t}$, respectively. We define the analogue to exceedance correlation, which we call exceedance Kendall's tau, as

$$
\begin{aligned}
& \tau^{+}(c)=\tau\left(U_{t}, V_{t} \mid U_{t}>c, V_{t}>c\right) \\
& \tau^{-}(c)=\tau\left(U_{t}, V_{t} \mid U_{t}<-c, V_{t}<-c\right) .
\end{aligned}
$$

In this paper, whenever we speak of $\tau^{+}$and $\tau^{-}$without specifying an exceedance level we mean $\tau(U, V \mid U>0.5, V>0.5)$ and $\tau(U, V \mid U<0.5, V<0.5)$. Thus they are the exceedance Kendall's tau for the exceedance level being the median of the distributions of $X$ and $Y$. The hypothesis of symmetric dependence we are interested in then becomes

$$
H_{0}: \quad \tau^{+}(c)=\tau^{-}(c), \quad \forall c \geq 0
$$

against

$$
H_{1}: \quad \tau^{+}(c) \neq \tau^{-}(c), \text { for some } c \geq 0
$$

In the next section we introduce a methods for testing the equality of the $\tau^{+}(c)$ and $\tau^{-}(c)$ for all $c$. 


\subsection{A Test Based on Mixtures of Copulas}

As before, let $F$ be the marginal distribution function of $X_{t}, G$ be the marginal distribution function of $Y_{t}$, and let $H$ be the joint distribution function of the pair of random variables $\left(X_{t}, Y_{t}\right)$. Then by the copula representation of multivariate distribution functions there exists a copula $C$ such that

$$
H(x, y)=C(F(x), G(y)), \forall(x, y) \in \overline{\mathbf{R}} \times \overline{\mathbf{R}},
$$

where $\overline{\mathbf{R}}$ denotes the extended real line. If $F$ and $G$ are continuous then $C$ is unique. Conversely if we have distribution functions $F$ and $G$ and a copula $C$, then $H$ is a bivariate distribution function. Recalling the probability integral transform for continuous distributions, which states that the random variable $U_{t}=F\left(X_{t}\right)$ has a $U(0,1)$ distribution regardless of the original distribution $F$, it becomes clear that a copula is no more than a multivariate distribution function with uniform marginals. It captures all the contemporaneous dependence between the random variables of interest, as all the dynamics of the marginal distributions are captured by $F$ and $G$ for $X_{t}$ and $Y_{t}$, respectively.

Kendall's tau between $X_{t}$ and $Y_{t}$ is determined by the underlying copula, which results from Theorem 5.1.3 in Nelson (2006). For $\left(U_{t}, V_{t}\right)=\left(F\left(X_{t}\right), G\left(Y_{t}\right)\right) \sim C_{\theta}\left(u_{t}, v_{t}\right)$ it boils down to the statement that

$$
\tau_{C}=4 \mathbb{E}\left(C_{\theta}\left(U_{t}, V_{t}\right)\right)-1
$$

where $\mathbb{E}$ denotes the expectation. For many copulas this expression can be solved to express $\tau$ in terms of the copula parameters. One parameter copulas can even be estimated by inverting the relationship between $\tau$ and the copula parameter $\theta$, resulting in a moment estimator. For a display of this method see Genest et al. (2005).

The conditional version of Kendall's tau can also be expressed in terms of the underlying copula.

Proposition 1 Let the joint distribution of $U_{t}$ and $V_{t}$ be $C_{\theta}\left(u_{t}, v_{t}\right)$. Then the population version of $\tau^{-}$defined in (10) for $c=0.5$ is determined by the underlying copula through

$$
\tau_{C}^{-}=\frac{\mathbb{E}\left[4 C_{\theta}\left(U_{t}, V_{t}\right)-2 C_{\theta}\left(U_{t}, 0.5\right)-2 C_{\theta}\left(0.5, V_{t}\right) \mid U_{t}<0.5, V_{t}<0.5\right]}{C_{\theta}(0.5,0.5)}+1
$$

The population version of $\tau^{+}$defined in (9) for $c=0.5$ is given by

$$
\tau_{C}^{+}=\frac{\mathbb{E}\left[4 C_{\theta}\left(U_{t}, V_{t}\right)-2 C_{\theta}\left(U_{t}, 0.5\right)-2 C_{\theta}\left(0.5, V_{t}\right)-1 \mid U_{t}>0.5, V_{t}>0.5\right]}{C_{\theta}(0.5,0.5)}+1
$$


A proof is given is given in the appendix. It is straightforward to derive formulas for exceedance levels different from 0.5. The conditional expectations can in general not be evaluated analytically. However, since it is computationally easy to simulate from most parametric classes of copulas one can evaluate these expectations numerically at any desired precision.

Two copulas that have received a lot of attention in the literature are the Gumbel and the Clayton copulas. Their distribution functions are given by

$$
C_{\theta}^{\text {Gumbel }}\left(u_{t}, v_{t}\right)=\exp \left(-\left[\left(-\ln \left(u_{t}\right)\right)^{\theta}+\left(-\ln \left(v_{t}\right)\right)^{\theta}\right]^{1 / \theta}\right)
$$

and

$$
C_{\theta}^{\text {Clayton }}\left(u_{t}, v_{t}\right)=\max \left[\left(u_{t}^{-\theta}+v_{t}^{-\theta}-1\right)^{\frac{-1}{\theta}}, 0\right] .
$$

They belong to the class of Archimedean copulas (see Nelson, 2006) and they are known to imply asymmetric dependence. In particular, they are characterized by tail dependence in one tail, and tail independence in the other (for a definition of tail dependence see Embrechts et al., 2003). Contour plots and simulated data of the two copulas with standard normal margins and parameters corresponding to Kendall's tau equal to 0.5 are provided in figure 1. Using the results from Proposition 1 we show that these two copulas imply asymmetric dependence. We evaluated the conditional expectations using 1000000 simulated data points and we let the parameters of the copulas vary corresponding to an overall Kendall's tau ranging from 0.01 to 0.99 . In figure $2 \tau^{+}$and $\tau^{-}$are plotted along with their difference. One can see that both copulas imply asymmetric dependence, but that the asymmetry is larger for the Clayton copula. Especially for lower overall dependence $\tau^{+}$is extremely small for the Clayton copula, which explains why often it not fit financial data too well, even though there is evidence of higher dependence for losses than for gains.

Given a copula $C\left(u_{t}, v_{t}\right)$ the corresponding survival, or rotated, copula is given by $C(1-$ $\left.u_{t}, 1-v_{t}\right)+u_{t}+v_{t}-1$. Using the fact that the convex combination of two copulas is again a copula two very flexible copulas are the Gumbel mixture (GM) and the Clayton mixture (CM) copulas given by

$$
C_{\alpha, \theta_{1}, \theta_{2}}^{G M}\left(u_{t}, v_{t}\right)=\alpha \cdot C_{\theta_{1}}^{\text {Gumbel }}\left(u_{t}, v_{t}\right)+(1-\alpha) \cdot\left(C_{\theta_{2}}^{\text {Gumbel }}\left(1-u_{t}, 1-v_{t}\right)+u_{t}+v_{t}-1\right)
$$

and

$$
C_{\alpha, \theta_{1}, \theta_{2}}^{C M}\left(u_{t}, v_{t}\right)=\alpha \cdot C_{\theta_{1}}^{\text {Clayton }}\left(u_{t}, v_{t}\right)+(1-\alpha) \cdot\left(C_{\theta_{2}}^{\text {Clayton }}\left(1-u_{t}, 1-v_{t}\right)+u_{t}+v_{t}-1\right) .
$$


Figure 1: Contour plots and simulated data from the Gumbel and Clayton Copula
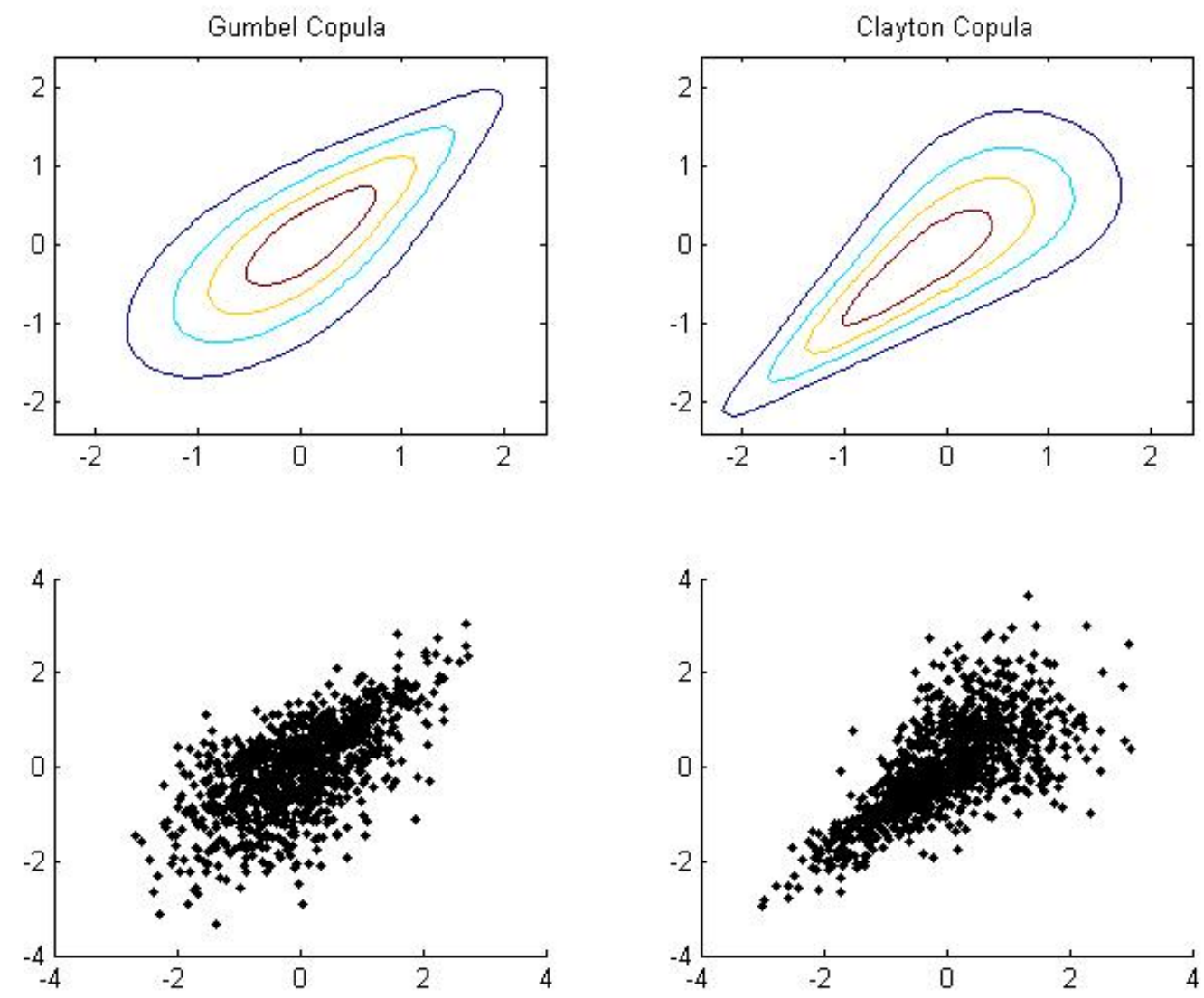

These Copulas nests symmetry when, jointly, $\alpha$ is equal to 0.5 and $\theta_{1}=\theta_{2}$.

We will use standard likelihood ratio tests to test the null hypothesis of symmetric dependence given in (11), which we base on the likelihood function for the models given in (19) and (20). We have to restrict $\alpha$ to be equal to 0.5 and additionally restrict $\theta_{1}$ to be equal to $\theta_{2}$, so we impose two restrictions. After estimating the restricted and unrestricted model our test statistic becomes

$$
L R_{C}=-2\left(L L_{r e s}-L L_{u r}\right) \sim \chi^{2}(2),
$$

where $L L_{r e s}$ is the log-likelihood of the restricted model, $L L_{u r}$ is the log-likelihood of the unrestricted model. Since we do not assume the copulas given in (19) and (20) to be the 
Figure 2: Conditional tau
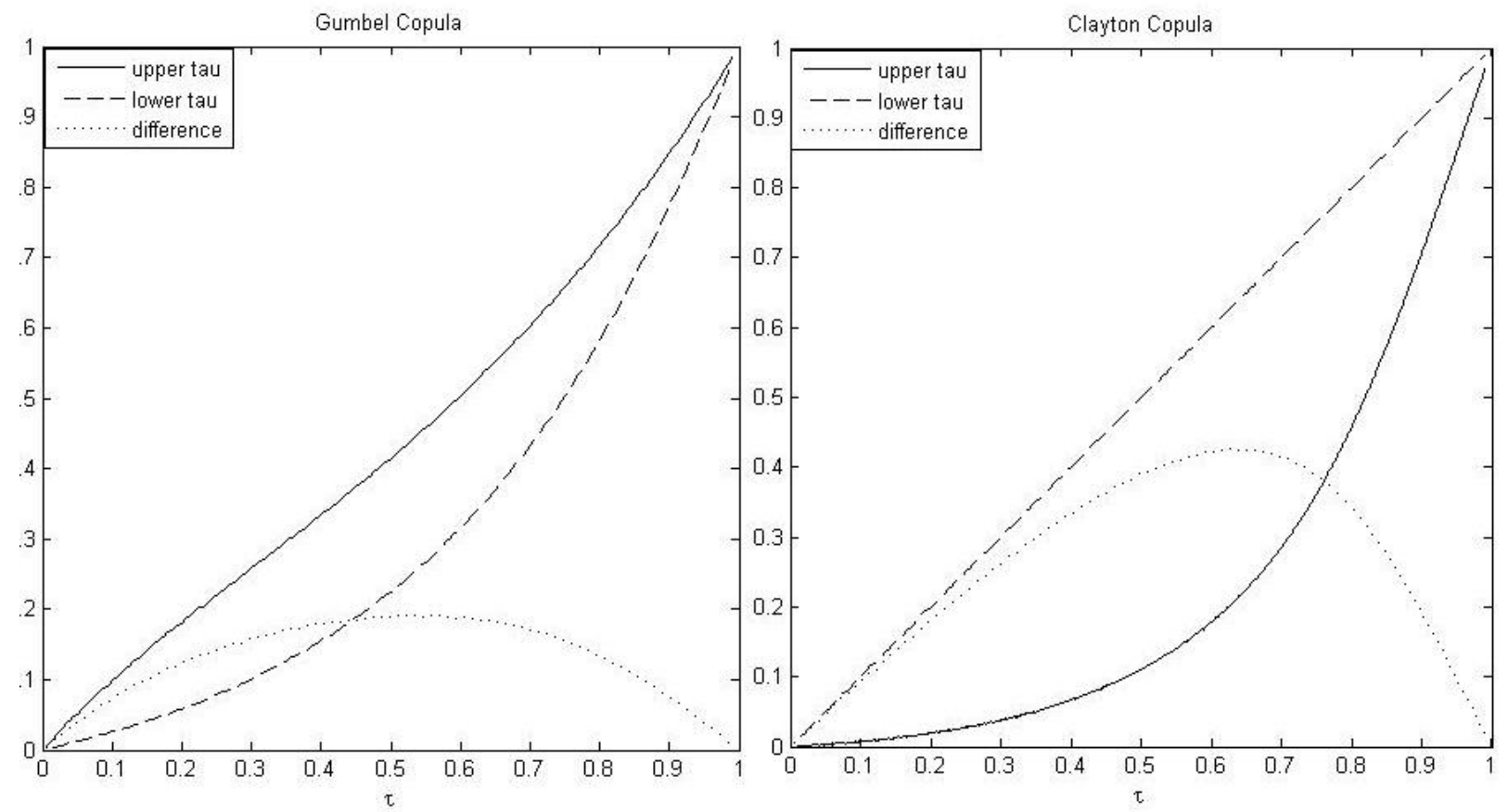

true ones this is in fact a pseudo LR test. From now on we denote the test based on the Gumbel mixture by GM-test and the one based on the Clayton mixture copula by CM-test. The use of these two copulas is motivated by the fact that the Clayton copula can capture very strong asymmetries. The Gumbel copula (or its survival version), on the other hand, tends to fit financial and economic data quite well in empirical applications.

In order to estimate one of the suggested copula models the variables of interest must be $U(0,1)$ distributed. Since we are not interested in the marginal distributions we can simply achieve that by using the empirical distribution function, which is given by

$$
\hat{F}(y)=\frac{1}{T} \sum_{t=1}^{T} \mathbf{1}_{\left\{Y_{t} \leq y\right\}} .
$$

This means that we are using the semi-parametric estimator studied in detail by Genest et al. (1995) for the i.i.d. case and by Chen and Fan (2006a,b) for time series.

There are two reason why we believe that our test is superior to the ones by Ang and Chen (2002) and Hong et al. (2007). First, as mentioned already Kendall's tau is a more robust measure of dependence and it is more appropriate when the data is not normally 8 
distributed, which is likely to be the case for most financial data. Second, when computing the two mentioned tests on may have to discard a large fraction of the data, in particular when the overall dependence is rather weak. This is not the case for the copula based test and thus its efficient use of the data available should lead to better size and power properties. This is confirmed by the Monte Carlo results below.

\section{How asymmetry is generated}

In this section we consider a few data generating processes (DGP) that imply asymmetric dependence even though the innovations were generated by a multivariate normal distribution. In the next section we perform Monte Carlo simulations to study these examples.

\section{Example 1: Bull and Bear Markets}

Let $R_{1 t}$ and $R_{2 t}$ be the returns in stock market 1 and 2 in period $t$. It is a stylized fact that stock markets have low volatility and correlation in periods of high returns (bull markets) and that volatility and correlation tend to increase when market have low (mostly negative) returns (bear markets). This corresponds to regime switching models for international equity markets such as the one considered in Ramchand and Susmel (1998). Consider the case that returns are generated by the following DGP in state $s=h, l$, where $h$ stands for bull state and $l$ for the bear state:

$$
\begin{gathered}
R_{1 t s}=\mu_{1 s}+\epsilon_{1 t s} \\
R_{2 t s}=\mu_{2 s}+\epsilon_{2 t s}
\end{gathered}
$$

where

$$
\left(\begin{array}{l}
\epsilon_{1 t s} \\
\epsilon_{2 t s}
\end{array}\right) \sim N\left[\left(\begin{array}{l}
0 \\
0
\end{array}\right),\left(\begin{array}{cc}
\sigma_{1 s}^{2} & \rho_{s} \sigma_{1 s} \sigma_{2 s} \\
\rho_{s} \sigma_{1 s} \sigma_{2 s} & \sigma_{2 s}^{2}
\end{array}\right)\right]
$$

If one ignores the state dependence of the distributions of $R_{1}$ and $R_{2}$ they will exhibit asymmetric dependence. As an example consider the simplified case that all standard deviations are equal to the mean return in the high state $\sigma_{1 h}=\sigma_{1 l}=\sigma_{2 h}=\sigma_{2 l}=\mu_{1 h}=\mu_{2 h}$, the mean returns in the bear state are minus the mean returns in the bull state $\mu_{1 l}=\mu_{2 l}=-\mu_{h}$, and the correlations are $\rho_{l}=0.3$ and $\rho_{h}=0.7$. Then the probabilities of observing joint exceedances of zero returns are $P\left(R_{1 l}>0, R_{2 l}>0\right)=0.084, P\left(R_{1 l}<0, R_{2 l}<0\right)=0.7667$, 
$P\left(R_{1 h}>0, R_{2 h}>0\right)=0.7281$, and $P\left(R_{1 h}<0, R_{2 h}<0\right)=0.0455$. Therefore the correlation between positive returns is dominated by the lower correlation during the bull state, whereas the correlation for negative returns is dominated by the higher correlation during the bear state, and together this implies asymmetric dependence. If additionally we assume that both state are equally likely we can calculate $\tau^{+}$and $\tau^{-}$for this setting using the formulas in Proposition 1. The corresponding copula is a mixture of two Gaussian copulas with $\rho_{1}=0.3$ and $\rho_{2}=0.7$, where the mixing parameter can be calculated using the exceedance probabilities calculated here. For $\tau^{+}$the weights are 0.9155 and 0.0845 and for calculating $\tau^{-}$they are 0.0691 and $0.9309 .{ }^{1} \tau^{-}$then is 0.2671 and $\tau^{+}$is 0.0997 .

\section{Example 2: Nonlinear Multivariate Models}

A second set of DGP's that may lead to the observation of asymmetric dependence are nonlinear multivariate models. Let the random variable $X$ and $Y$ be generated as follows

$$
\begin{aligned}
& X_{t}=\delta I\left(\epsilon_{2 t}>c\right)+\epsilon_{1 t} \\
& Y_{t}=\delta I\left(\epsilon_{1 t}>c\right)+\epsilon_{2 t} .
\end{aligned}
$$

$\epsilon_{1 t}$ and $\epsilon_{2 t}$ are, possibly correlated, $N(0,1)$ variables. In general, dynamics should be allowed for and the model could be a nonlinear VAR, but for pedagogical purposes we stick to this simplified version for now. In the simulation study, however, we do allow for dynamics. This is similar to the model for stock market contagion considered by Pesaran and Pick (2007). Consider regressing the $X$ and $Y$ on an intercept and collecting the residuals, which then are tested for asymmetric dependence. The population version of the linearized model is

$$
\begin{aligned}
& X_{t}=\delta P\left(\epsilon_{2 t}>c\right)+\epsilon_{1 t}^{*} \\
& Y_{t}=\delta P\left(\epsilon_{1 t}>c\right)+\epsilon_{2 t}^{*} .
\end{aligned}
$$

Denote $P\left(\epsilon_{1 t}>c\right)=P\left(\epsilon_{2 t}>c\right) \equiv a$. Then the mean of the linearized model is $a \delta$ and the relation between the errors of the linearized model and the true errors is

$$
\begin{gathered}
\epsilon_{1 t}^{*}=\epsilon_{1 t}+\delta I\left(\epsilon_{2 t}>c\right)-a \delta \\
\epsilon_{2 t}^{*}=\epsilon_{2 t}+\delta I\left(\epsilon_{1 t}>c\right)-a \delta .
\end{gathered}
$$

Due to the nonlinear nature of the DGP we cannot calculate the $\tau^{+}$and $\tau^{-}$directly. However, we can get an intuitive idea how asymmetry is generated for this DGP. To do so we

\footnotetext{
${ }^{1}$ The weights are found such that the probabilities of exceedance for the mixing copula are equal to the ones found in the example. Using the CDF of the Gaussian copula at $(0.5,0.5)$ for $\rho$ equal to 0.3 and 0.7 this is straightforward.
} 
must distinguish the cases where $c>a \delta$ and $c<a \delta$. For the second case the situation with $c=0$ is the most important one.

Case 1: $c>a \delta$

In this situation the probabilities that the errors of the linearized model jointly lie below and above zero can be shown to be

$$
\begin{aligned}
& P\left(\epsilon_{1 t}^{*}<0, \epsilon_{2 t}^{*}<0\right)=P\left(\epsilon_{1 t}<a \delta, \epsilon_{2 t}<a \delta\right) \\
& P\left(\epsilon_{1 t}^{*}>0, \epsilon_{2 t}^{*}>0\right)=P\left(\epsilon_{1 t}>a \delta, \epsilon_{2 t}>a \delta\right)+2 P\left(a-1<\epsilon_{1 t}<a \delta, \epsilon_{2 t}>c\right) .
\end{aligned}
$$

Clearly, the $\tau^{-}$is not affected by threshold effects, because all of the original errors that generate these observation jointly lie below the thresholds. The $\tau^{+}$on, the other hand, is affected, but it is a bit delicate to see in what way. The first part of $P\left(\epsilon_{1 t}^{*}>0, \epsilon_{2 t}^{*}>0\right)$ can be decomposed into those original errors that jointly exceed the threshold and those that do not. Once both errors exceed the threshold, the errors from the linearized model jump by the value $\delta$. A second pair of observations from the original errors for which only one variable exceeds the threshold is now more likely of being concordant with this first pair than before, since one of the components may have been larger than the corresponding one from the first pair. After the effect of the jump this may not be the case anymore.

It is unclear in what way concordance is affected for the terms in the second part of the probability. We expect the effect to be more or less neutral.

Case 2: $c<a \delta$

Now the probabilities that the residuals of the linearized model jointly lie below and above zero are

$$
\begin{aligned}
& P\left(\epsilon_{1 t}^{*}<0, \epsilon_{2 t}^{*}<0\right)=P\left(\epsilon_{1 t}<c, \epsilon_{2 t}<c\right) \\
& P\left(\epsilon_{1 t}^{*}>0, \epsilon_{2 t}^{*}>0\right)=P\left(\epsilon_{1 t}>c, \epsilon_{2 t}>c\right)+2 P\left(a-1<\epsilon_{1 t}<c, \epsilon_{2 t}>c\right) .
\end{aligned}
$$

The effect of the threshold will be constant when the original errors are both above the threshold and the effect described in case 1 cannot occur anymore. Thus we expect the asymmetry generated in this case to be rather small.

In figure 3 we present a scatterplot of data generated from the this model with $\delta=0.7$ and correlation of 0.3 between the errors for $c=0$ and $c=0.5$. For a better exposition the variables have been mapped into $U(0,1)$ variables by the empirical probability integral 
Figure 3: Simulated data for example 2
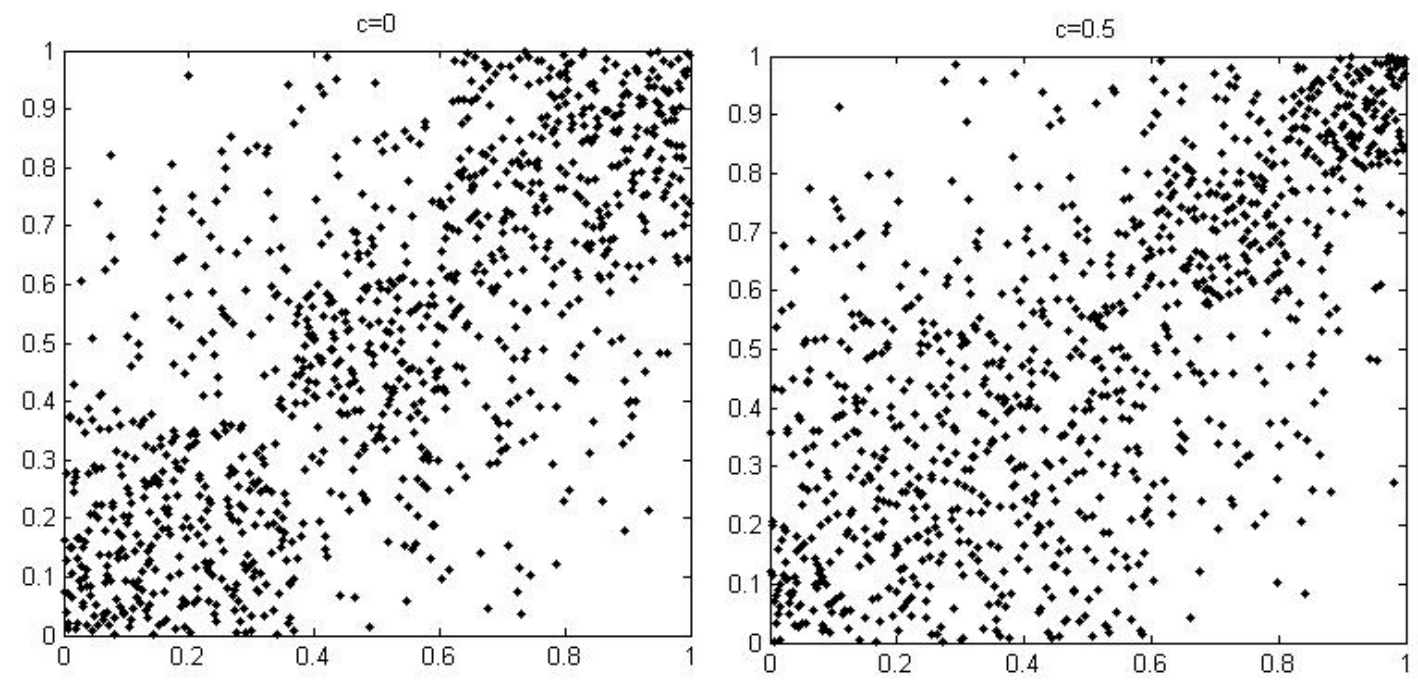

transform. For $c=0.5$ the asymmetry is quite apparent and is the data looks similar to observations generated by a Gumbel or a survival Clayton copula. In the plot for $c=0$ the dependence seems to be rather symmetric.

Finally, we evaluated the $\tau^{+}$and $\tau^{-}$by Monte Carlo simulation. For $c=0$ they are 0.2393 and 0.2379 , whereas for $c=0.5$ they turn out to be 0.3622 and 0.0896 , respectively. Thus the conclusions of our informal argument above are confirmed.

\section{Example 3: Nonlinear Autoregressions}

Copulas cannot only be used to model the contemporaneous dependence between i.i.d. random variables, but they may be used to model time dependence of a single variable. Instead of describing the dependence between $X_{t}$ and $Y_{t}$ one can fit a copula to $Y_{t}$ and $Y_{t-1}$, thus generalizing the normal AR(1) model for stationary variables and treating the random process $Y_{t}$ as a stationary first order Markov chain. More generally, a copula of dimension $p$ can be used to model autoregressive processes of order $p-1 .^{2}$ These models are called Copula Markov models. For details on these models see Joe (1997) or Bouyé et al. (2001). Chen and Fan (2006a) establish the validity of the semi-parametric estimation approach to this type of models when $Y_{t}$ is a stationary Markov chain of order 1, namely they present conditions

\footnotetext{
${ }^{2}$ The bivariate margins of $(i, i+1)$ need to be restricted to be the same for all $i$.
} 
for processes generated by this type of models to be $\beta$-mixing and they establish consistency and asymptotic normality. Furthermore, they suggest an estimator for the asymptotic variance of the estimator. These results justify the validity of applying our tests for symmetric dependence on $Y_{t}$ and $Y_{t-1}$ whenever $Y_{t}$ can be treated as a stationary first order Markov chain and the asymptotic $\chi^{2}$ distribution of the likelihood ratio test statistic remains appropriate. In case the dynamics of a time series of interest are best described by a Gaussian copula and the margins are also normal, meaning linear dependence is appropriate, we are just replicating a linear AR model. However, in case the dependence is of an asymmetric type, a linear AR model may not be an appropriate model for the dynamics present in the data anymore. Conversely, data generated by a nonlinear autoregressive process is likely to be show asymmetric dependence. The example we consider here is the class of smooth transition autoregressions (STAR). For a survey on STAR models see Dijk et al. (2002). Thus in the model of interest $Y_{t}$ is generated by

$$
Y_{t}=\left(\alpha_{1}+\beta_{1} Y_{t-1}\right)\left(1-G\left(s_{t} ; \gamma, c\right)\right)+\left(\alpha_{2}+\beta_{2} Y_{t-1}\right) G\left(s_{t} ; \gamma, c\right)+\varepsilon_{t},
$$

where popular choice for $G$ is the logistic function given by

$$
G\left(s_{t} ; \gamma, c\right)=\left(1+\exp \left\{-\gamma\left(s_{t}-c\right)\right\}\right)^{-1},
$$

with $s_{t}$ being a state variable, $c$ the threshold value and $\gamma$ the smoothness parameter. When $s_{t}=Y_{t-d}(29)$ is called self-exiting STAR or SETAR and this is the model we consider here with $d=1$.

An advantage of using the copula based tests for the null of symmetric time dependence is that although the corresponding Copula Markov models are in general non-linear and asymmetric they nest symmetry as a special case. Besides we do not have to deal with problems of unidentified parameters in the logistic function under the null hypothesis like the classical tests for STAR models relying on Taylor expansions.

It is rather obvious that the simple first order STAR generates asymmetric dependence between $Y_{t}$ and $Y_{t-1}$ as long as $\beta_{1} \neq \beta_{2}$. In figure 3 we illustrate the degree of asymmetry generated by this DGP in terms of the difference between the $\tau^{+}$and $\tau^{-}$. The parameter choices are $\alpha_{1}=\alpha_{2}=0, \beta_{1}=0.4, c=1$ and $\gamma=2.5$. $\beta_{2}$ ranges from 0.4 to 0.9 and $\varepsilon_{t} \sim N(0,1)$. One can see that a significant degree of asymmetry is present even for small differences between $\beta_{1}$ and $\beta_{2}$.

\section{Monte Carlo Studies}

In this section we provide an extensive Monte Carlo study to assess the quality of the copula based tests for asymmetric dependence. In the first subsection we study the size and power 
Figure 4: Difference between $\tau^{+}$and $\tau^{-}$for data generated by a SETAR model

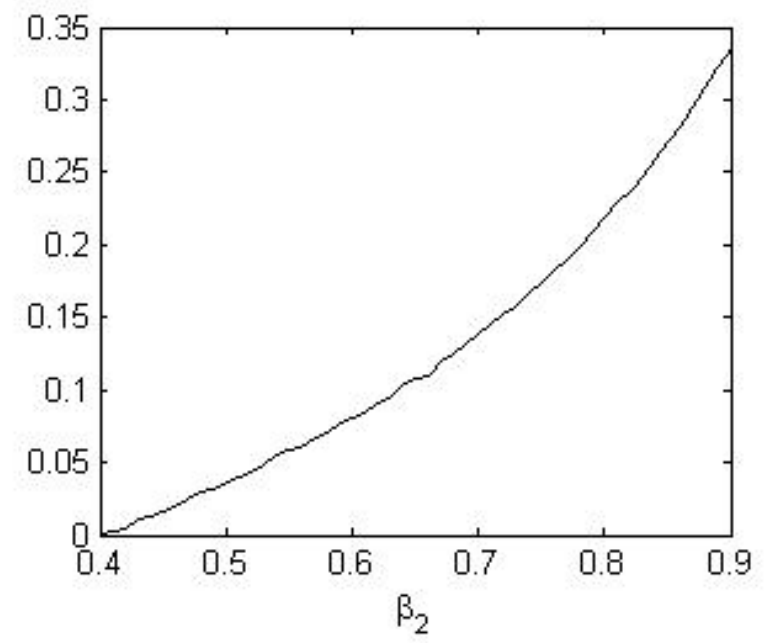

of the tests and compare it to the tests of Hong et al. (2007). In the other three subsections we study the behavior of the copula based tests for the three cases studied in section 3 .

\subsection{The General Case}

In order to study the properties of the Gumbel mixture (GM) and Clayton mixture (CM) tests in comparison to the test for asymmetric correlation proposed by Hong et al. (2007), which we label $J$-test, we chose a data generating process that is quite similar to the ones used by these authors in their simulation. ${ }^{3}$ We perform the tests for the null hypothesis of symmetric dependence on i.i.d. $U(0,1)$ data generated by the following copula model.

$$
C(u, v)=\alpha \cdot C_{\theta_{1}}^{\text {Clayton }}(u, v)+(1-\alpha) \cdot C_{\theta_{2}}^{\text {Normal }}(u, v)
$$

$\theta_{1}$ and $\theta_{2}$ are chosen such that the overall kendall's tau of the mixture is equal to 0.5. This DGP is characterized by symmetric dependence when $\alpha=0$, but asymmetric dependence increases as $\alpha$ get larger. We let the mixing parameter $\alpha$ take on the values $(0,0.25,0.5$, $0.75,1)$ and we consider 4 different sample sizes, namely $(100,250,500,1000)$. The rejection frequencies based on 1000 Monte Carlo simulations are reported in table 1 . All the tests perform well in terms of size, although the GM-test is a bit undersized. The power of the

\footnotetext{
${ }^{3}$ Hong et al. (2007) demonstrated in their paper that the $J$-test is superior to the $H^{2}-$ test proposed by Ang and Chen (2002), so we do not consider it here.
} 
Table 1: Rejection frequency of the null of symmetric dependence for data generated by (30)

\begin{tabular}{|c|c|c|c|c|c|c|c|}
\hline$n=100$ & J-test & Gumtest & Claytest & $\mathrm{n}=250$ & J-test & Gumtest & Claytest \\
\hline$\alpha=0$ & 0.048 & 0.021 & 0.039 & & 0.042 & 0.019 & 0.045 \\
\hline$\alpha=0.25$ & 0.079 & 0.091 & 0.128 & & 0.116 & 0.226 & 0.241 \\
\hline$\alpha=0.5$ & 0.183 & 0.315 & 0.404 & & 0.384 & 0.795 & 0.817 \\
\hline$\alpha=0.75$ & 0.386 & 0.747 & 0.789 & & 0.73 & 0.995 & 0.992 \\
\hline$\alpha=1$ & 0.62 & 0.971 & 0.965 & & 0.964 & 1 & 1 \\
\hline $\mathrm{n}=500$ & & & & $n=1000$ & & & \\
\hline$\alpha=0$ & 0.035 & 0.024 & 0.035 & & 0.029 & 0.028 & 0.046 \\
\hline$\alpha=0.25$ & 0.205 & 0.455 & 0.489 & & 0.388 & 0.789 & 0.793 \\
\hline$\alpha=0.5$ & 0.672 & 0.986 & 0.977 & & 0.938 & 1 & 1 \\
\hline$\alpha=0.75$ & 0.959 & 1 & 1 & & 0.999 & 1 & 1 \\
\hline$\alpha=1$ & 1 & 1 & 1 & & 1 & 1 & 1 \\
\hline
\end{tabular}

Note: Table 1 reports the rejection frequency of the null hypothesis symmetric dependence using the test by Hong et al. (2007) and two copula based tests. The DGPs is a mixture of a Normal and a Clayton copula with parameters corresponding to an overall kendall's tau of 0.5 . The case $\alpha=0$ corresponds to the size of the tests. The number of Monte Carlo replications is fixed to 1.000.

tests, however, is always significantly higher for the copula based tests, especially for smaller sample sizes and degrees of asymmetry. The CM-test stands out as the best performing one with power often twice that of the $J$-test. This comes as no surprise, as the data were partly generated by a Clayton copula. However, when we replace the Clayton copula in the DGP by the Gumbel copula the $G M$ - test and the $C M$ - test are about the same in terms of power ${ }^{4}$, so the higher flexibility of the Clayton copula, indicated by the larger attainable asymmetry that can be seen in figure 2, may increase its usefulness for the problem at hand. As mentioned above, the reason for the copula based tests to outperform the $J$-test is most likely that they rely on a more robust measure of dependence and that these tests use the full information of the sample, whereas the $J$-test only uses those observations that are jointly above and below the exceedance levels. Note that the J-test has been performed on the $U(0,1)$ variables, so it actually tests the symmetry of the rank correlation coefficient also known as Spearman's $\rho$. When we transformed the data into $N(0,1)$ variables the J-test is severely undersized (below 1\%) and has lower power. The copula based tests circumvent this problem by automatically standardizing the data with the empirical probability integral transform.

\footnotetext{
${ }^{4}$ The results of this alternative simulation are available upon request.
} 


\subsection{Bull and Bear Markets}

Data is generated from the bull and bear markets model (23). We consider two different cases, one where volatility remains constant across states and one where it increases during the bear state. The mean is chosen to be the same for both markets, namely $\mu_{h}=0.01$ and $\mu_{l}=-0.01$. The standard deviation $\sigma_{s}$ is equal to 0.01 . In the case that it increases during the bear state it is equal to 0.02 . The change in regime always occurs at $\mathrm{T} / 2$, where $\mathrm{T}$ is the sample size, which takes on the values $(100,250,500,1000)$. Finally, $\rho_{l}$ is chosen to be 0.3 , whereas $\rho_{h}$ varies across the values $(0.4,0.5,0.6,0.7)$. Before applying our tests we transform

Table 2: Rejection frequency of the null of symmetric dependence for data generated by (23)

\begin{tabular}{lrrrr}
\hline \hline$\sigma_{h}=0.01$ & $\sigma_{l}=0.01$ & & & \\
GM-test & $\rho_{h}=0.4$ & $\rho_{h}=0.5$ & $\rho_{h}=0.6$ & $\rho_{h}=0.7$ \\
$\mathrm{~T}=100$ & 0.027 & 0.073 & 0.2 & 0.379 \\
$\mathrm{~T}=250$ & 0.035 & 0.135 & 0.447 & 0.809 \\
$\mathrm{~T}=500$ & 0.071 & 0.331 & 0.784 & 0.985 \\
$\mathrm{~T}=1000$ & 0.117 & 0.611 & 0.984 & 1 \\
\hline $\mathrm{CM}-$ test & & & & \\
$\mathrm{T}=100$ & 0.058 & 0.11 & 0.231 & 0.485 \\
$\mathrm{~T}=250$ & 0.063 & 0.249 & 0.537 & 0.873 \\
$\mathrm{~T}=500$ & 0.103 & 0.452 & 0.86 & 0.989 \\
$\mathrm{~T}=1000$ & 0.188 & 0.737 & 0.994 & 1 \\
\hline$\sigma_{h}=0.01$ & $\sigma_{l}=0.02$ & & & \\
$\mathrm{GM}-\mathrm{test}$ & $\rho_{h}=0.4$ & $\rho_{h}=0.5$ & $\rho_{h}=0.6$ & $\rho_{h}=0.7$ \\
$\mathrm{~T}=100$ & 0.165 & 0.23 & 0.362 & 0.51 \\
$\mathrm{~T}=250$ & 0.404 & 0.581 & 0.783 & 0.915 \\
$\mathrm{~T}=500$ & 0.744 & 0.897 & 0.979 & 0.999 \\
$\mathrm{~T}=1000$ & 0.975 & 0.999 & 1 & 1 \\
\hline $\mathrm{CM}-\mathrm{test}$ & & & & \\
$\mathrm{T}=100$ & 0.301 & 0.357 & 0.486 & 0.637 \\
$\mathrm{~T}=250$ & 0.586 & 0.724 & 0.858 & 0.96 \\
$\mathrm{~T}=500$ & 0.867 & 0.953 & 0.985 & 1 \\
$\mathrm{~T}=1000$ & 0.991 & 1 & 1 & 1 \\
\hline \hline
\end{tabular}

Note: Table 2 reports the rejection frequency of the null hypothesis symmetric dependence using the two copula based tests. The data has been generated by (23) with $\mu_{h}=0.01$ and $\mu_{l}=-0.01$. The change in regime always occurs at $T / 2$. The number of Monte Carlo replications is fixed to 1.000 . 
the two artificially generated return series into $U(0,1)$ variables by the empirical probability integral transform. Table (2) shows that the two tests are able to identity the asymmetric dependence reasonably well, as long as the change in correlation is large enough. Again the CM-test slightly outperforms the GM-test in all situations. It is notable that a change in variance additionally to a change in correlation strongly increases the asymmetry. This can be explained by the reasoning of Forbes and Rigobon (2002) that changes in volatility lead to spurious increases in observed correlation combined with our calculations in section 3 .

\subsection{Nonlinear VAR}

We extend the DGP (24) by introducing an intercept and some dynamics, so that it becomes the following simple nonlinear vector autoregression $(\mathrm{VAR})^{5}$

$$
\begin{aligned}
& X_{t}=\alpha_{1}+\beta_{1} X_{t-1}+\beta_{2} Y_{t-1}+\delta I\left(\epsilon_{2 t}>c\right)+\epsilon_{1 t} \\
& Y_{t}=\alpha_{2}+\gamma_{1} X_{t-1}+\gamma_{2} Y_{t-1}+\delta I\left(\epsilon_{1 t}>c\right)+\epsilon_{2 t} .
\end{aligned}
$$

The parameter choices are $\alpha_{1}=0.2, \alpha_{2}=0.1, \beta_{1}=0.3, \beta_{2}=0.1, \gamma_{1}=0.1$ and $\gamma_{2}=0.3$. The innovations are drawn from a standard multivariate normal distribution with correlation coefficient equal to 0.5. The threshold value $c$ takes on the values $(0,0.5,1,1.5)$, the effect of the threshold $\delta$ varies over $(0.3,0.5,0.7,0.9)$ and the sample sizes we consider are $(100$, $250,500,1000)$. A linear $\operatorname{VAR}(1)$ is fit to the data and the residuals are collected. After transforming them into $U(0,1)$ variables by the empirical probability integral transform the two copula based tests are performed. As before, the number of Monte Carlo replications is equal to 1000 . The results can be found in table 3 . The rejection frequency of the null of symmetric dependence increases with $\delta$ and with the sample size. It also depends on $c$ and is highest for $c=1$. That makes intuitively sense, as a larger threshold means that less observations exceed it and thus the effect of the indicator function occurs less often. Even after the introduction of intercepts and dynamics it still holds that when $c=0$, the DGP hardly causes any asymmetric dependence, confirming that the findings in example 2 still hold after the DGP is generalized. Finally, the CM-test again outperforms the GM-test in terms of power.

\subsection{SETAR Models}

In this final Monte Carlo simulation we illustrate how the tests for asymmetric dependence can be used to distinguish nonlinear autoregressive processes from linear ones. We generate

\footnotetext{
${ }^{5}$ Strictly speaking this is a linear VAR, as the nonlinearity only enters through the innovations, which are martingale differences. As mentioned before, the DGP is comparable to the model suggested by Pesaran and Pick (2007), but it is easier to analyze.
} 
Table 3: Rejection frequency of the null of symmetric dependence for data generated by (31)

\begin{tabular}{lrrrrrrrr}
\hline \hline CM-test & & & \multicolumn{7}{c}{ GM-test } \\
$\mathrm{T}=100$ & $\mathrm{c}=0$ & $\mathrm{c}=0.5$ & $\mathrm{c}=1$ & $\mathrm{c}=1.5$ & $\mathrm{c}=0$ & $\mathrm{c}=0.5$ & $\mathrm{c}=1$ & $\mathrm{c}=1.5$ \\
$\delta=0.3$ & 0.068 & 0.141 & 0.201 & 0.128 & 0.036 & 0.083 & 0.116 & 0.07 \\
$\delta=0.5$ & 0.092 & 0.332 & 0.449 & 0.248 & 0.035 & 0.233 & 0.325 & 0.175 \\
$\delta=0.7$ & 0.085 & 0.518 & 0.694 & 0.405 & 0.04 & 0.405 & 0.649 & 0.347 \\
$\delta=0.9$ & 0.084 & 0.662 & 0.893 & 0.55 & 0.055 & 0.56 & 0.815 & 0.441 \\
\hline $\mathrm{T}=250$ & & & & & & & & \\
$\delta=0.3$ & 0.067 & 0.255 & 0.399 & 0.207 & 0.026 & 0.178 & 0.351 & 0.231 \\
$\delta=0.5$ & 0.084 & 0.614 & 0.855 & 0.514 & 0.028 & 0.522 & 0.823 & 0.518 \\
$\delta=0.7$ & 0.088 & 0.876 & 0.986 & 0.794 & 0.044 & 0.762 & 0.976 & 0.812 \\
$\delta=0.9$ & 0.094 & 0.971 & 0.999 & 0.924 & 0.043 & 0.935 & 0.997 & 0.933 \\
\hline $\mathrm{T}=500$ & & & & & & & & \\
$\delta=0.3$ & 0.066 & 0.467 & 0.734 & 0.409 & 0.029 & 0.365 & 0.648 & 0.408 \\
$\delta=0.5$ & 0.108 & 0.894 & 0.991 & 0.838 & 0.029 & 0.82 & 0.984 & 0.824 \\
$\delta=0.7$ & 0.107 & 0.992 & 1 & 0.984 & 0.039 & 0.975 & 1 & 0.988 \\
$\delta=0.9$ & 0.091 & 1 & 1 & 1 & 0.038 & 0.999 & 1 & 0.999 \\
\hline $\mathrm{T}=1000$ & & & & & & & & \\
$\delta=0.3$ & 0.061 & 0.753 & 0.943 & 0.715 & 0.032 & 0.683 & 0.933 & 0.718 \\
$\delta=0.5$ & 0.085 & 0.997 & 1 & 0.993 & 0.037 & 0.989 & 1 & 0.996 \\
$\delta=0.7$ & 0.089 & 1 & 1 & 1 & 0.035 & 1 & 1 & 1 \\
$\delta=0.9$ & 0.088 & 1 & 1 & 1 & 0.039 & 1 & 1 & 1 \\
\hline \hline
\end{tabular}

Note: Table 3 reports the rejection frequency of the null hypothesis symmetric dependence using the two copula based tests. The data has been generated by (31) with $\alpha_{1}=0.2, \alpha_{2}=0.1, \beta_{1}=0.3, \beta_{2}=0.1$, $\gamma_{1}=0.1$ and $\gamma_{2}=0.3$. The number of Monte Carlo replications is fixed to 1.000. 
a time series from DGP (29). The intercept $\alpha$ is chosen equal to $0.2, \beta_{1}=0.4$ and the innovations come from a standard normal distribution. We vary both the parameter $\beta_{2}$, which can take on the values $(0.5,0.6,0.7)$, the threshold $c$ (values are 0,1 and 2 ) and the smoothness parameter $\gamma$ of the logistic function, which is varies over $(1,2.5,3.5,10)$. The series $Y$ is transformed into a standard uniform variable and the asymmetry tests are applied to $Y_{t}$ and $Y_{t-1}$. For spatial reasons we report the outcomes, which can be found in table 4, only for the case of 1000 observations. The tests are able to capture the nonlinearities reasonably well. The size of the tests (not reported) is about the same as for the general case reported above. As a comparison we also performed a test based on a third order Taylor expansions that was designed for logistic STAR models in the same simulation setup. The results, which are available upon request, showed that the latter test has about the same power as the GM-test and hence performs worse than the CM-test.

Table 4: Rejection frequency of the null of symmetric dependence for data generated by (29)

\begin{tabular}{rrrrrrrrr}
\hline \hline CM-test & & \multicolumn{7}{c}{ GM-test } \\
$\beta_{2}=0.5$ & $\gamma=1$ & $\gamma=2.5$ & $\gamma=3.5$ & $\gamma=10$ & $\gamma=1$ & $\gamma=2.5$ & $\gamma=3.5$ & $\gamma=10$ \\
$c=0$ & 0.115 & 0.136 & 0.141 & 0.135 & 0.103 & 0.095 & 0.102 & 0.1 \\
$c=1$ & 0.106 & 0.196 & 0.2 & 0.201 & 0.078 & 0.147 & 0.157 & 0.158 \\
$c=2$ & 0.097 & 0.148 & 0.142 & 0.166 & 0.08 & 0.119 & 0.104 & 0.14 \\
\hline$\beta_{2}=0.6$ & & & & & & & & \\
$c=0$ & 0.416 & 0.488 & 0.462 & 0.423 & 0.38 & 0.416 & 0.403 & 0.38 \\
$c=1$ & 0.455 & 0.674 & 0.706 & 0.688 & 0.402 & 0.615 & 0.644 & 0.632 \\
$c=2$ & 0.319 & 0.544 & 0.578 & 0.607 & 0.275 & 0.515 & 0.546 & 0.579 \\
\hline$\beta_{2}=0.7$ & & & & & & & & \\
$c=0$ & 0.829 & 0.823 & 0.806 & 0.749 & 0.789 & 0.781 & 0.778 & 0.71 \\
$c=1$ & 0.858 & 0.966 & 0.972 & 0.969 & 0.831 & 0.961 & 0.953 & 0.95 \\
$c=2$ & 0.713 & 0.934 & 0.949 & 0.975 & 0.68 & 0.923 & 0.94 & 0.974 \\
\hline \hline
\end{tabular}

Note: Table 4 reports the rejection frequency of the null hypothesis symmetric dependence between $Y_{t}$ and $Y_{t-1}$ using the two copula based tests. The data has been generated by (29) with $\alpha=0.2$ and $\beta_{1}=0.4$. The number of observations is equal 1000 to and the number of Monte Carlo replications is fixed to 1.000.

\section{Empirical Applications}

In the section we apply the tests for asymmetric dependence two different data set. First we test for asymmetric dependence between monthly returns of 17 international stock markets, 
Table 5: Application on international stock market returns

\begin{tabular}{lrrrr}
\hline \hline & GM-test & CM-test & avg. $\hat{\tau}^{+}$ & avg. $\hat{\tau}^{-}$ \\
Mean rejection and con. $\tau$ & 0.6838 & 0.6912 & 0.1459 & 0.2434 \\
\hline \hline
\end{tabular}

Note: Table 5 columns 2 and 3 report the fraction of pairs of stock market returns for which the null of symmetric dependence is rejected at a 5\% confidence level using the GM- and CM-test. Columns 4 and 5 report the average measure of $\tau^{+}$and $\tau^{-}$.

which relates to example 1 in section 3. Second the tests are applied on macroeconomic data. The tests are used to test both for asymmetric dependence over time of the individual series as suggested in example 3 and for asymmetric dependence of the residuals of a linear and nonlinear VAR motivated by example 2 .

\subsection{International sock market returns}

The first data set we consider are monthly stock market returns for the most important stock markets in the world over a period of over 30 years spanning the period from January 1974 to December 2006. This amount to a total of 396 observations per series and a total of 136 pairs of countries. ${ }^{6}$ The data has been obtained from Datastream. The two tests we have introduced are applied each of the 136 pairs of stock market returns and the fraction of rejections of the null hypothesis of symmetric dependence at a $5 \%$ confidence level is reported in table 5 together with average estimates of $\tau^{+}$and $\tau^{-}$. The outcomes do not only suggest that the majority of pairs is characterized by asymmetric dependence, but also that the dependence is higher for negative returns that it is for positive returns. These findings do not come as a surprise and similar ones have been documented in the literature mentioned in the introduction, as well as in many other studies using copulas or conditional correlation measures. Thus we consider a further field of application of our tests.

\subsection{Application on US output and unemployment data}

The data set we consider to illustrate our testing procedure are quarterly observations of US GNP at 1982 prices and total unemployment ranging from 1951 until 1990. In order to have stationary data the log difference has been taken of the output series and the first difference of the unemployment series, leading to a total of 156 observations. The data have been used by Altissimo and Violante (2001), who modeled them with a threshold VAR.

\footnotetext{
${ }^{6}$ The countries we consider are Australia, Austria, Belgium, Canada, Denmark, France, Germany, Hong Kong, Ireland, Italy, Japan, Netherlands, South Africa, Singapore, Switzerland, UK and USA.
} 
Table 6: Tests for asymmetric dependence on the autoregressive dynamics of Unemployment and GNP

\begin{tabular}{lrrrr}
\hline \hline & GM-test & CM-test & $\hat{\tau}^{+}$ & $\hat{\tau}^{-}$ \\
Change in unemployment & 0.014 & 0.0085 & 0.3631 & 0.0727 \\
Change in log GNP & 0.7227 & 0.6034 & 0.2549 & 0.113 \\
\hline \hline
\end{tabular}

Note: Table 6 reports the p-values of the null hypothesis symmetric dependence and the sample estimates of $\tau^{+}$and $\tau^{-}$between the observations at time $t$ and time $t-1$ for the change in unemployment and change in log GNP using data from the US ranging from 1951:Q1 to 1990:Q4.

The first step of our analysis is testing for asymmetries in the autoregressive dynamics of the two individual series. In a preliminary analysis AR models have been fit to the data to check the order of the dynamics present. This is an important step, as our approach only is valid if $Y_{t}$ can be treated as a first order Markov process. Ignoring dependencies with higher lags could severely influence the results. For both series only the first order autoregressive term was significant, which means we do not have to worry about omitted higher order dynamics when using copulas to mimic the $\mathrm{AR}(1)$ process. The data have been transformed into $U(0,1)$ variables using the empirical probability integral transform and the tests for symmetry are applied on the transformed variables $\hat{u}_{t}$ and $\hat{u}_{t-1}$. Table 6 presents the $\mathrm{p}$-values of the tests along with the sample estimates of $\tau^{+}$and $\tau^{-}$. Symmetric dependence is rejected for the unemployment series at a $5 \%$ confident level. For the change in output we cannot reject the null of symmetric dependence. This means that when modeling the two series by $\mathrm{AR}(1)$ models the change in output can be well described by a linear model, whereas the change in unemployment series should be modeled by a non-linear specification such as the STAR model. The findings for the GNP are in contrast to Potter (1995) who, using a slightly different sample period, included more lags in the autoregressive specification (most of which were not significant), and who found evidence for a Self-Exciting Threshold Autoregression.

Next, we test for asymmetric dependence of the residuals of a VAR model for the two series. Following Altissimo and Violante (2001) we chose a lag length of 2 to capture the dynamics. We consider two specifications of the VAR. The first one is the standard linear one, whereas the second one is the threshold VAR specification that was used by Altissimo and Violante (2001). For the nonlinear specification the VAR is augmented with 2 lags of the following feedback variable

$$
C D R_{t}(r, k)=y_{t}-\max \left(y_{t}, y_{t-1}+r, \ldots, y_{t-k}+r\right),
$$

where $y$ is the real GNP. The variable captures periods where output is lower than the maximum in past periods increased by a threshold $r$, so it identifies recession periods. The 
Table 7: Tests for asymmetric dependence on the residuals of a linear and nonlinear VAR

\begin{tabular}{lrrrr}
\hline \hline & GM-test & CM-test & $\hat{\tau}^{+}$ & $\hat{\tau}^{-}$ \\
linear VAR & 0.0047 & 0 & 0.0316 & 0.3643 \\
nonlinear VAR & 0.3227 & 0.2712 & 0.0118 & -0.0165 \\
\hline \hline
\end{tabular}

Note: Table 7 reports the p-values of the null hypothesis symmetric dependence and the sample estimates of $\tau^{+}$and $\tau^{-}$between the residuals of a linear $\operatorname{VAR}(2)$ and a $\operatorname{VAR}(2)$ augmented with two lags of the variable CDR defined in (32) for the change in unemployment and change in log GNP using data from the US ranging from 1951:Q1 to 1990:Q4.

threshold was determined endogenously by Altissimo and Violante (2001) and turned out to be -0.138 and we set $k=10$. Table 7 reports the outcomes of the tests and the sample estimates of $\tau^{+}$and $\tau^{-}$. For the residuals of the linear VAR we clearly reject the null of symmetric dependence. When the threshold variable is included this is not the case anymore. Overall these results are in line with Altissimo and Violante (2001). They found that nonlinear dynamics are only present in the output equation and that the nonlinearity of the unemployment series "transmits to output purely through its cross correlation". This finding can also explain the difference of our specification to the one by Potter (1995).

\section{Conclusion}

Different kinds of asymmetries and nonlinearities are frequently observed in economic and financial data. These may be hard to distinguish from one another in a given data set. In this paper we have proposed a way to test for a specific type of nonlinearity, namely asymmetric dependence structures. We defined a conditional version of Kendall's tau as the probability of concordance minus the probability of discordance of a pair of random variables conditional on both variables being above or below their respective medians. This adjusted dependence measure serves as a basis for our definition and tests of asymmetric dependence based either on the Gumbel or the Clayton copula. The conditional dependence structures of these two copulas are analyzed more carefully than has been done in previous studies. The tests we propose have good size properties and clearly outperform existing approaches for the same problem in terms of power.

Different data generating processes may lead to the observation of asymmetric dependencies. This highlights an important issue: What do I know if I reject symmetric dependence? The answer depends on the situation at hand and can not be generally answered. Often it can be a sign of misspecification of an underlying model such as ignoring different regimes, structural breaks, time varying parameters, changing volatilities and leverage effects. In 
other cases it may point to a feature in the data that cannot be easily explained, but that should nevertheless be regarded when e.g. making investment decisions. In any case it tells us that a linear approximation may not capture the important aspects of the data in a satisfactory way. This may mean that linear correlation is not the appropriate measure of (unconditional) stock market dependence, that the autoregressive dynamics in the change in unemployment are not sufficiently captured by a linear AR model or that a linear VAR is not enough to capture the joint dynamics of the changes in output and unemployment.

\section{A Proof of Proposition 1}

We start by proving the general formula

$$
\tau_{C}=4 \mathbb{E}(C(U, V))-1
$$

Let $(U, V) \sim C$, where $C$ is a Copula. Take an arbitrary pair $(u, v)$ drawn from Copula $C$. We are interested in the probability that a second pair $\left(u^{\prime}, v^{\prime}\right)$ drawn from $C$ is concordant with the first pair minus the probability that the two pairs are discordant, i.e. in the quantity $P[(u-U)(v-V)>0]-P[(u-U)(v-V)<0]$. Dividing the unit square into four regions we have concordance for points in the rectangles southwest (which we call A) and northeast $(\mathrm{C}$ ) of $(u, v)$ and discordance in the rectangles southeast (B) and northwest (D) of $(u, v)$. The probability of an observation in each of the rectangles is given by its H-Volume (see Nelson, 2007 for a definition):

$$
\begin{aligned}
& P(A)=C(u, v)+C(0,0)-C(u, 0)-C(0, v) \\
& P(C)=C(1,1)+C(u, v)-C(u, 1)-C(1, v) \\
& P(B)=C(1, v)+C(u, 0)-C(1,0)-C(u, v) \\
& P(D)=C(u, 1)+C(0, v)-C(0,1)-C(u, v)
\end{aligned}
$$

The probability of concordance minus the probability of discordance is equal to $P(A)+$ $P(C)-P(B)-P(D)$. Noting the basic properties that a copula is grounded $(C(a, 0)=$ $C(0, a)=0$ for any $a)$ and that it has margins $(C(b, 1)=C(1, b)=b$ for any $b)$ we find that

$$
P(A)+P(C)-P(B)-P(D)=4 C(u, v)-2 v-2 u+1 .
$$

Integrating this expression over all possible points $(u, v)$ over the unit square with respect to the underlying copula and noting that the mean of a standard uniform random variable is 0.5 the formula in (33) follows. 
Now take an arbitrary pair $(u, v)$ such that $u>0.5$ and $v>0.5$. The joint probability that a second pair $\left(u^{\prime}, v^{\prime}\right)$ from $C$ is concordant with $(u, v)$ and that both coordinates are greater than 0.5 minus the joint probability that the second point is discordant with $(u, v)$ and both coordinates are greater than 0.5, i.e. $P[(u-U)(v-V)>0 \mid U, V>0.5]-P[(u-U)(v-V)<$ $0 \mid U, V>0.5]$, is given by

$$
C(u, v)-2 C(u, 0.5)-2 C(0.5, v)-2 u-2 v+2+C(0.5,0.5)
$$

Note that the probability that both coordinates of a pair from $C$ are larger than $0.5, P[u>$ $0.5, v>0.5]$, is equal to $C(0.5,0.5)$ and the conditional probability is the joint probability divided by the marginal probability. Then the conditional probability of concordance minus the conditional probability discordance is equation (34) divided by $C(0.5,0.5)$. Integrating this over all points $(u, v)$ such that $u>0.5$ and $v>0.5$ and noting that for a $U(0,1)$ variable $E(U \mid U>0.5)=0.75$, the expression for $\tau^{+}$follows.

The formula for $\tau^{-}$is derived in a similar way and its derivation is omitted. 


\section{References}

[1] Altissimo, F. and Giovanni L. Violante (2001), "The non-linear dynamics of output and unemployment in the U.S", Journal of Applied Econometrics, 16(4), 461-486.

[2] Ang, A. and J. Chen (2002), "Asymmetric Correlations of Equity portfolios", Journal of Financial Economics, 63, 443-494.

[3] Bouyé, E., N. Gaussel and M. Salmon (2001), "Investigating Dynamic Dependence Using Copulae", Working Paper, Financial Econometrics Research Centre, Sir John Cass Business School, London.

[4] Candelon, B. and H. Manner (2007) "Testing for Asset Market Linkages: A new Approach based on Time-Varying Copulas", METEOR Research Momorandum, $\mathrm{RM} / 07 / 052$

[5] Cappiello, L., R. Engle and K. Sheppard (2007) "Asymmetric Dynamics in the Correlations of Global Equity and and Bond Returns", Journal of Financial Econometrics, $4,537-572$.

[6] Cappiello, L., B. Gérard and S. Manganelli (2005) "Measuring Comovements by Regression Quantiles", ECB Working Paper.

[7] Chen, X. and Y. Fan (2006a), "Semiparametric Estimation of Copula-Based Time Series Models", Journal of Econometrics, 130, 307-335.

[8] Chen, X. and Y. Fan (2006b), "Estimation and Model Selection of Semiparametric Copula-Based Multivariate Dynamic Models Under Copula Misspecification", Journal of Econometrics, vol. 135, p.125-154.

[9] Van Dijk, D., T. Tersvirta and P.H. Franses (2002), "Smooth Transition Autoregressive Models - A Survey of recent developments", Econometric Reviews 21, 1-47.

[10] Embrechts, P., A. McNeil and D. Straumann (2002), "Correlation and Dependence Properties in Risk Management: Properties and Pitfalls", in M. Dempster, ed., Risk Management: Value at Risk and Beyond, Cambridge University Press.

[11] Embrechts, P., F. Lindskog and A. McNeil (2003), "Modelling Dependence with Copulas and Applications to Risk Management", in Handbook of Heavy Tailed Distributions in Finance, Elsevier, Chapter 8, 329-384. 
[12] Forbes, K.J., Rigobon, R. (2002), "No Contagion, only Interdependence: measuring Stock Market Co-movements", Journal of Finance, 57 (5), 2223-2261.

[13] Genest, C., K. Ghoudi and L.-P. Rivest (1995), " A semiparametric estimation procedure of dependence parameters in multivariate families of distributions", Biometrika, 82, 543552.

[14] Genest, C., R.W.J. Goorbergh and B.J.M. Werker (2005), "Multivariate Options Pricing Using Dynamic Copula Models", Insurance: Mathematics and Economics, 37, 101-114.

[15] Hong, Y, J. Tu and G. Zhou (2007), "Asymmetries in Stock Returns: Statistical Tests and Economic Evaluation", Review of Financial Studies, 20, 1547-1581.

[16] Joe, H. (1997), Multivariate Models and Dependence Concepts, Chapman \& Hall/CRC.

[17] King, M. and S. Wadhwani (1990), "Transmission of Volatility Between Stock Markets", Review of Financial Studies, 3, 3-33.

[18] Longin, F. and B. Solnik (1995), "Is the Correlation in International Equity Returns Constant: 1960-1990", Journal of International Money and Finance, 14, 3-26.

[19] Longin, F. and B. Solnik (2001), "Extreme Correlation of International Equity Markets", Journal of Finance, 56, 649-676.

[20] Nelsen, R.B. (2006), An Introduction to Copulas, Springer Verlag, Berlin.

[21] Newey, W. and K. West (1994), "Automatic Lag Selection in Covariance Matrix Estimation", Review of Economic Studies, 61, 631-653.

[22] Patton, A. (2006), "Modelling Asymmetric Exchange Rate Dependence", International Economic Review, 47(2), 527-556.

[23] Pesaran H. and Pick, A., (2007) "Econometric Issues in the Analysis of Contagion", Journal of Economic Dynamics and Control 31, 1245-1277.

[24] Potter, S.M. (1995), "A Nonlinear Approach to US GNP", Journal of Applied Econometrics, 10, 109-125

[25] Ramchand L. and Susmel, R. (1998), "Volatility and Cross Correlation across Major Stock Markets", Journal of Empirical Finance, 5, 397-416. 